\title{
A pseudoaneurysmal cyst in the pulmonary artery secondary to lung suppuration
}

Hideo Ichimura, MD, ${ }^{a}$ Hiroo Okazaki, MD, ${ }^{a}$ Kazunori Kikuchi, MD, Toshihiko Saitoh, MD, ${ }^{\mathrm{c}}$ Norihiro Haraguchi, MD, ${ }^{\mathrm{c}}$ and Masataka Onizuka, MD, ${ }^{\mathrm{d}}$ Ibaraki, Japan

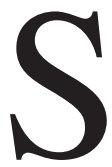

ince the time that antibiotics have come into common use, pseudoaneurysms in the pulmonary artery resulting from infectious conditions have been extremely rare. ${ }^{1}$ We herein present the case of a patient with familial diabetes mellitus, who had lung suppuration with a pseudoaneurysmal cyst in his pulmonary artery. Only use of enhanced (as opposed to plain) computed tomography (CT) could provide us with the clue that led us to presume this rare condition as a provisional diagnosis prior to surgery.

\section{Clinical Summary}

A 53-year-old Japanese man, who is a current smoker with poorly controlled familial diabetes mellitus, had 1-week common cold-like symptoms and right chest pain without seeking medical attention. When his fever rose to above $38^{\circ} \mathrm{C}$, he went to a hospital. Based on plain CT findings (Figure 1, A), lung suppuration was suspected and the patient was sent to another hospital for further evaluation and treatment. Although only alpha streptococcus (gram-positive cocci) and neisseria (gramnegative cocci) were detected by sputum culture, it is noteworthy that a gram-stained specimen of sputum showed three types of microbes, namely gram-positive cocci, gram-negative cocci, and gram-negative rod. Sputum culture revealed alpha streptococcus and neisseria. One-week administration of the ampicillin sodium/sulbactam sodium and clindamycin failed to bring about an improvement, and an enhanced chest CT was performed, which showed exacerbation of the lung suppuration manifested by the appearance of parapneumonic effusion and increased gas formation resulting in expansion of the middle lobe. It also revealed a round mass in the consolidated right middle lobe, which was enhanced equally to the pulmonary vessel (Figure $1, B$ ). A pulmonary artery aneurysm secondary to lung suppuration was suspected, and the patient was transferred to our hospital. We performed a right thoracotomy, which revealed that the inflamed right middle lobe was grayish and necrotic (Figure 2, A). We gently dissected the right mid-

\footnotetext{
From the Departments of General Thoracic Surgery ${ }^{\mathrm{a}}$ and Pathology, Tsukuba Medical Center Hospital; Department of Pulmonary Medicine, Tsukuba Gakuen Hospital; and Department of Surgery, ${ }^{\mathrm{d}}$ Institute of Clinical Medicine, University of Tsukuba, Ibaraki, Japan.

Address for reprints: Hideo Ichimura, MD, Department of General Thoracic Surgery, Tsukuba Medical Center Hospital, 1-3-1 Amakubo, Tsukuba-shi, Ibaraki 305-8558, Japan (E-mail: ichimura@tmch.or.jp).

J Thorac Cardiovasc Surg 2007;133:1678-9

$0022-5223 / \$ 32.00$

Copyright @ 2007 by The American Association for Thoracic Surgery doi:10.1016/j.jtcvs.2007.01.072
}

dle lobe of the lung and exposed the middle pulmonary artery and vein. We severed the middle pulmonary artery prior to the vein to avoid rupturing the aneurysmal structure on the assumption that the blood flow was communicated into the lesion. All ge-

latinous and fibrinous tissue and localized effusion were evacuated from the pleural space. When we cut across the removed middle lobe, darkish blood poured out from the cavitary lesion. Moreover, the probe revealed the connection between the cavity and the pulmonary artery (Figure 2, B). Pathologically, the cavitary lesion was mainly composed of granulation tissue and was not covered with the endothelium; therefore, this case was diagnosed as a pseudoaneurysmal cyst secondary to lung sup-
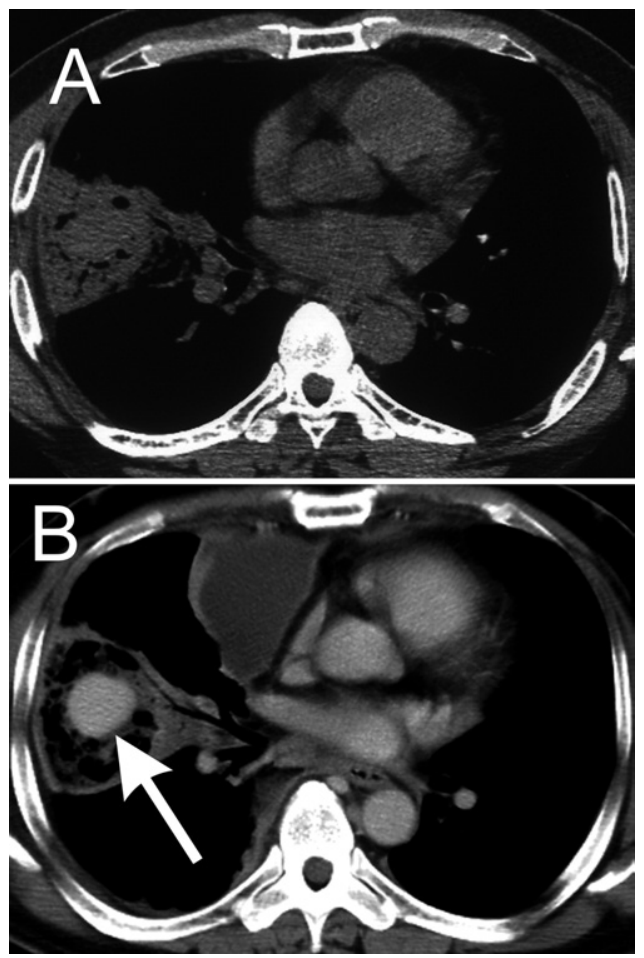

Figure 1. A, Plain chest CT showed consolidation in the middle lobe with unstructural gas formation. B, Enhanced CT taken after 1-week administration of antibiotics clearly showed a round lesion enhanced equally to the pulmonary vessel (arrow). 


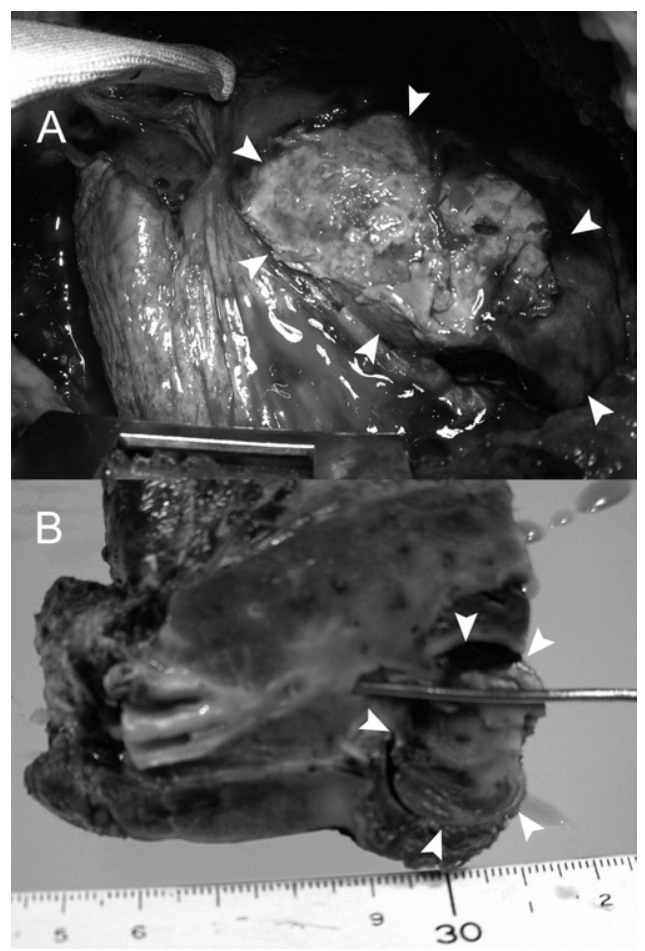

Figure 2. A, Intraoperative photograph showed a grayish, pyogenic right middle lobe (surrounded by arrow heads). B, A probe of the cut surface of the resected specimen revealed a connection between the cavitary lumen (the cavitary margin is denoted by arrowheads) and the pulmonary artery.

puration. Pathologic examination showed gram-positive cocci in the cavity and cavitary wall, and bacteriologic culture and polymerase chain reaction for the acid-fast bacillus of the cavitary content were all negative. The patient's postoperative course was uneventful, and he was discharged in good condition and remains asymptomatic a year after surgery.

\section{Discussion}

In this patient, there had been no indication of an abnormal chest shadow at his annual health examinations. Therefore, we have ruled out the possibility of a preexisting pulmonary vessel abnormality or pulmonary lesion that could have been a predispositional factor leading to this lethal condition. Although in the literature not all the reported cases had pathologic confirmation of whether aneurysmal lesions were covered by endothelium, in cases reported as both pulmonary artery aneurysm and pseudoaneurysm, infectious causes were varied, including tuberculosis, syphilis, mucormycosis, aspergilosis, candida, Staphylococcus aureus, and streptococcus species. $^{2}$ Other acquired etiologies are iatrogenic Swan-Ganz catheter insertion, trauma, and tumor associated. ${ }^{3-5}$ In our case, an excessive inflammation due to pyogenic agents resulted in a breakdown of the pulmonary artery leading to the formation of the pseudoaneurysmal cyst. Some may suggest that pulmonary artery coil embolization is an option that would make operation safer $^{3}$; however, we speculate that it may be a hindrance, especially when severing the pulmonary artery. Although the lack of improvement with the administration of antibiotics justifies a thoracotomy, we believe that the cautious removal of the aneurysmal lesion in the pyogenic lung parenchyma was the best option. It is important to note that the clinical course and plain CT led to an operative indication but they did not uncover the aneurysmal lesion. Only enhanced CT gave us detailed enough information so that we could approach the latent nature of the lesion prior to the surgery.

\section{References}

1. Chung CW, Doherty JU, Kotler R, et al. Pulmonary artery aneurysm presenting as a lung mass. Chest. 1995;108:1164-6.

2. Bowler RP, Durham J, Schwarz MI. Massive hemoptysis from a pulmonary artery aneurysm associated with an emphysematous bulla. Chest. 1998;113:1130-1.

3. Dimarakis I, Thorpe JA, Papagiannopoulos K. Successful treatment of a posttraumatic pulmonary artery pseudoaneurysm with coil embolization. Ann Thorac Surg. 2005;79:2134-6.

4. Oliver TB, Stevenson AJ, Gillespie IN. Pulmonary artery pseudoaneurysm due to bronchial carcinoma. Br J Radiol. 1997;0:950-1.

5. Poplausky MR, Rozenblit G, Rundback JH, et al. Swan-Ganz catheterinduced pulmonary artery pseudoaneurysm formation: three case reports and a review of the literature. Chest. 2001;120:2105-11. 